\title{
In vivo dosimetry and acute toxicity in breast cancer patients undergoing intraoperative radiotherapy as boost
}

\author{
Jason Joon Bock Lee, MD', Jinhyun Choi, MD1', Sung Gwe Ahn, MD², Joon Jeong, MD², \\ Ik Jae Lee, MD¹, Kwangwoo Park, PhD¹, Kangpyo Kim, MD1, Jun Won Kim, MD¹ \\ Departments of 'Radiation Oncology and ${ }^{2}$ Surgery, Gangnam Severance Hospital, \\ Yonsei University College of Medicine, Seoul, Korea
}

Purpose: To report the results of a correlation analysis of skin dose assessed by in vivo dosimetry and the incidence of acute toxicity. This is a phase 2 trial evaluating the feasibility of intraoperative radiotherapy (IORT) as a boost for breast cancer patients. Materials and Methods: Eligible patients were treated with IORT of $20 \mathrm{~Gy}$ followed by whole breast irradiation (WBI) of 46 Gy. A total of 55 patients with a minimum follow-up of 1 month after WBI were evaluated. Optically stimulated luminescence dosimeter (OSLD) detected radiation dose delivered to the skin during IORT. Acute toxicity was recorded according to the Common Terminology Criteria for Adverse Events v4.0. Clinical parameters were correlated with seroma formation and maximum skin dose. Results: Median follow-up after IORT was 25.9 weeks (range, 12.7 to 50.3 weeks). Prior to WBI, only one patient developed acute toxicity. Following WBI, 30 patients experienced grade 1 skin toxicity and three patients had grade 2 skin toxicity. Skin dose during IORT exceeded $5 \mathrm{~Gy}$ in two patients: with grade 2 complications around the surgical scar in one patient who received $8.42 \mathrm{~Gy}$. Breast volume on preoperative images ( $p=0.001)$, ratio of applicator diameter and breast volume $(p=0.002)$, and distance between skin and tumor ( $p=0.003)$ showed significant correlations with maximum skin dose.

Conclusions: IORT as a boost was well-tolerated among Korean women without severe acute complication. In vivo dosimetry with OSLD can help ensure safe delivery of IORT as a boost.

Keywords: Boost with intraoperative radiotherapy, Segmental mastectomy, Toxicity, In vivo dosimetry

\section{Introduction}

Whole breast irradiation (WBI) followed by tumor bed boost is standard radiation treatment in patients with breast cancer undergoing breast-conserving surgery (BCS). Nevertheless, in selected patients with early breast cancer, partial breast irradiation (PBI) has shown acceptable local control rates with good cosmetic results [1]. Targeted intraoperative radiotherapy-A (TARGIT-A) trial used low energy $\mathrm{X}$-rays ( $50 \mathrm{kV}$ ) generated from Intrabeam (Carl Zeiss Meditec AG, Oberkochen, Germany) for delivering PBI during BCS and reported noninferiority of intraoperative radiotherapy (IORT) compared to WBI using external beam radiation therapy (EBRT) in selected early breast cancer patients [2]. While American Society for Radiation Oncology (ASTRO) consensus guidelines recommend accelerated PBI for selected patients with early breast cancer [3], the feasibility of applying IORT as a boost to WBI in highrisk patients remains to be demonstrated. The use of IORT

Received 23 March 2017, Revised 27 April 2017, Accepted 22 May 2017.

Correspondence: Jun Won Kim, MD, PhD, Department of Radiation Oncology, Gangnam Severance Hospital, Yonsei University College of Medicine, 211 Eonju-ro, Gangnam-gu, Seoul 06273, Korea. Tel: +82-2-2019-3154, Fax: +82-2-2019-4855, E-mail: junwon@yuhs.ac

(c) This is an Open Access article distributed under the terms of the Creative Commons Attribution Non-Commercial License (http://creativecommons.org/ licenses/by-nc/4.0/) which permits unrestricted non-commercial use, distribution, and reproduction in any medium, provided the original work is properly cited.

www.e-roj.org 
as boost has been examined in retrospective studies $[4,5]$ and a randomized comparison of these techniques with WBI followed by EBRT boost is currently underway in the TARGIT-B trial.

IORT delivers high dose to the tumor cavity and spares normal breast tissue; however, the radiation dose delivered to overlying skin and association with toxicity require close attention. Acute and late complications of IORT using Intrabeam include pain, seroma, wound infection, fibrosis, hyperpigmentation, edema of the breast, and ulceration $[6,7]$. One limitation of IORT is the lack of image-guided pretreatment plans, without which operators are unable to anticipate the radiation dose delivered to adjacent organs such as the overlying skin. Therefore, methods to assess radiation dose to the skin during IORT delivery (in vivo dosimetry) can be helpful in predicting the risk of developing skin toxicities and establishing proper inclusion criteria for future IORT applications. Since applying IORT as a boost has the potential to result in increased skin toxicity compared with IORT alone, additional safety evaluation is required for Korean patients whose breast volumes tend to be smaller than those of Western population. Since August 2014, a phase 2 trial investigating safety and feasibility of IORT used as a boost treatment in Korean patients requiring BCS has been underway at our institution. In the present report, we present the results of in vivo dosimetry and the correlation analysis of the factors associated with increased skin dose and acute toxicity.

\section{Materials and Methods}

\section{Study endpoints and eligibility}

The primary endpoint was to provide an independent assessment of the delivered dose and consistency of the IORT technique through optically stimulated luminescent dosimeter (OSLD)-based in vivo dosimetry. The secondary endpoint was to determine clinical factors associated with increased risk of delivering a higher skin dose than intended. The phase 2 trial allowed enrollment of patients with histologically proven breast cancer undergoing BCS, aged 20 years or older, and with maximum tumor size less than $5 \mathrm{~cm}$ and Eastern Cooperative Oncology Group (ECOG) performance status of 1 or 2, but excluded patients who received neoadjuvant chemotherapy prior to $\mathrm{BCS}$, or who required surgery for bilateral breast cancer. The study protocol conforms to the ethical guidelines of the 1975 Declaration of Helsinki, and was approved by Gangnam Severance Hospital Institutional Review Board (No. 3-2013-0299). All subjects provided informed consent prior to

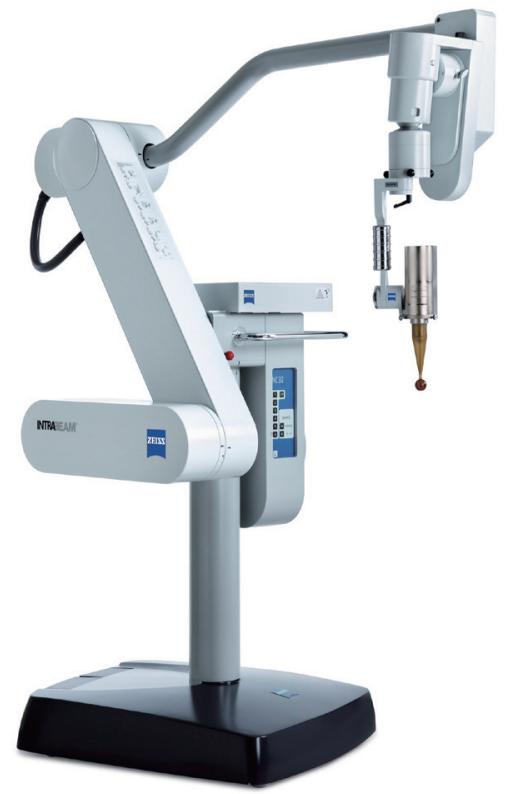

Fig. 1. Intraoperative radiotherapy device.

enrollment; all patients presented at a multidisciplinary tumor board at our institution.

\section{IORT procedure}

Two experienced surgeons were in charge of BCS. During BCS, a single fraction of $20 \mathrm{~Gy}$ was prescribed to the surface of the tumor cavity using the mobile 50-kV X-ray source (Intrabeam) (Fig. 1). The $X$-ray source was attached to the robotic arm which maintained the stability of the source throughout the whole process. Isotropy and the output of the unit were verified; the pre-IORT calibration process required by the system was performed prior to each treatment. Immediately after excision of the tumor, frozen sections were sent to the Department of Pathology for analysis to prevent positive resection margins after BCS. Re-excision was performed in cases of positive resection margins on frozen section analysis prior to IORT. A spherical applicator with an appropriate diameter (ranging from 1.5 to $5.0 \mathrm{~cm}$ in $0.5 \mathrm{~cm}$ increments) was selected according to the size of the tumor cavity, and the applicator was attached over the probe of the X-ray source. With a sterile sheath draped over the IORT device to prevent contamination, the spherical applicator was placed inside the tumor cavity, and a purse-string suture was used to pull the walls of the tumor cavity tightly against the applicator surface. The edges of the skin incision were everted so any part of skin was at least $1 \mathrm{~cm}$ away from the applicator surface to avoid excessive radiation exposure. When the skin-to-tumor distance 
was less than $1 \mathrm{~cm}$, the portion of the skin overlying the tumor was excised to prevent suboptimal radiation dose delivery to the high risk area due to skin-edge eversion. Actual beamon time after radiation site shielding was 20 to 30 minutes depending on applicator diameter. The surface of the tumor cavity received $20 \mathrm{~Gy}$, while the radiation dose was attenuated to approximately $5 \mathrm{~Gy}$ at $1 \mathrm{~cm}$ depth. IORT was followed, with or without chemotherapy, by WBI of 46 Gy in 23 fractions.

\section{In vivo dosimetry procedure}

The OSLD used was InLight nanoDots (Landauer Inc., Glenwood, IL, USA) which has a $5 \mathrm{~mm}$ diameter with thickness of $0.2 \mathrm{~mm}$ protected in a plastic casing. For each IORT measurement, 8 OSLD chips were annealed and attached to the skin, at $5 \mathrm{~mm}$ and $15 \mathrm{~mm}$ from the skin edge in the superior, inferior, medial and lateral locations (Fig. 2). After IORT, an InLight microStar reader (Landauer Inc.) which utilizes a photomultiplier tube (PMT) to collect the luminescence of the OSLD was used to determine the results of in vivo dosimetry. Each OSLD chip underwent measurements 3 times in the microStar reader; the average PMT count was recorded.

\section{Toxicity evaluation and breast volume measurement}

The Common Terminology Criteria for Adverse Events v4.0 was used for toxicity assessment. Findings such as wound infection, edema, erythema and palpable seroma were documented 1 week and 1 month after IORT, at the end of WBI, and 1 month after WBI. Volumes of aspirated seroma were also documented. The ipsilateral breast of each patient on preoperative chest computed tomography images in supine position and breast magnetic resonance imaging (MRI) images in prone position was contoured according to the Radiation Therapy Oncology Group (RTOG) Breast Cancer Atlas for Radiation Therapy Planning. MIMVISTA 6.3.3. (MIM Software Inc., Cleveland, $\mathrm{OH}_{1}$ USA) was used for contouring and calculating the preoperative breast volume of each patient on both imaging modalities.

\section{Statistical analysis}

Variables correlated with the maximum skin dose during IORT were determined using linear regression analysis; factors associated with seroma requiring aspiration 1 month after IORT were evaluated using logistic regression analysis. These variables included applicator diameter, skin-to-tumor distance in preoperative images, maximum tumor diameter, breast volume, volume of hemi-breast containing the tumor, ratio of applicator diameter and breast volume, and ratio of applicator volume and breast volume. The ratio of applicator diameter

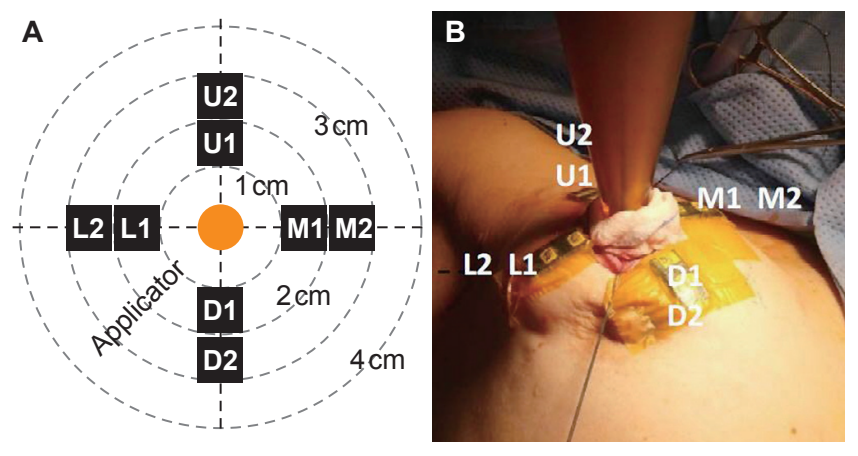

Fig. 2. (A) Schematic arrangement of optically stimulated luminescent dosimeter (OSLD) and (B) example of OSLD arrangement in operating room.

and breast volume was calculated as

$$
\frac{\text { Applicator diameter }(\mathrm{cm})}{\text { Breast volume }(\mathrm{mL}) / 1,000}
$$

and the ratio of applicator volume and breast volume was calculated as

$$
\frac{\text { Applicator volume }(\mathrm{mL})}{\text { Breast volume }(\mathrm{mL}) / 1,000} \text {. }
$$

The $p$-values of $<0.05$ were considered significant; all statistical analyses were performed using IBM SPSS ver. 20.0 (SPSS Inc., Chicago, IL, USA).

\section{Results}

\section{Patients and treatment characteristics}

From August 2014 until August 2015, IORT was considered in 124 patients; 82 patients actually underwent IORT and were enrolled in the phase 2 study. Among the 42 patients who did not undergo IORT, the reasons of IORT cancellation were either preoperative decisions in multidisciplinary conference, including presence of a huge primary mass or multicentric tumor nodules $(n=4)$; neoadjuvant chemotherapy $(n=3)$; definitive axillary, supraclavicular, or internal mammary lymph node metastasis $(n=3)$; and tumor close to the skin ( $n=$ 9); and intraoperative decisions including persistent positive resection margins ( $n=9$ ), conversion to total mastectomy ( $n$ $=8)$, and shape of tumor cavity unsuitable for IORT ( $n=6)$. Among the 82 patients treated with IORT, 55 patients who underwent a minimum follow-up period of 1 month after WBI were included in the analysis. Follow-up after IORT ranged from 12.7 weeks to 50.3 weeks with a median follow-up of 
25.9 weeks. The median patient age was 52 years (range, 32 to 69 years), invasive ductal carcinoma was the most common type (69.1\%), and tumors were most frequently located in the upper quadrant (87.3\%). T stages according to the American Joint Committee on Cancer (AJCC) 7th edition were Tis in 7 patients, $\mathrm{T} 1$ in 40 patients and $\mathrm{T} 2$ in 8 patients; 4 patients were staged with $\mathrm{N} 1$ disease after operation with a single positive axillary lymph node. No patient had positive margins in frozen sections, but permanent pathology revealed positive margins in 3 patients. In these 3 patients, surgical re-excision was recommended; however, the patients refused. Sixteen patients received adjuvant chemotherapy and 37 patients received hormonal therapy. The median interval between IORT and of the initiation of EBRT was 5.7 weeks (range, 4.4 to 29.1 weeks). Patient, tumor, and treatment characteristics are summarized in Table 1. The median beam-on time was 18.15 minutes (range, 17.25 to 26.27 minutes); the diameters of applicators used were $2.5 \mathrm{~cm}(\mathrm{n}=3), 3.0 \mathrm{~cm}(\mathrm{n}=19), 3.5 \mathrm{~cm}(\mathrm{n}=28)$, and 4.0 $\mathrm{cm}(\mathrm{n}=5)$.

\section{Toxicity}

IORT followed by WBI was well tolerated. Although one patient experienced wound infection and required wound revision after BCS and IORT, no other patient experienced acute complications between IORT and the 1-month follow-up. Adverse skin reactions following WBI were observed in $33(60 \%)$ patients, among whom 3 (5.5\%) experienced grade 2 toxicity. More severe toxicity was not reported (Table 2). Fifty patients with seroma required aspiration at 1 week after IORT, 28 at 1 month after IORT, and 10 immediately after WBI.

\section{In vivo dosimetry}

At $5 \mathrm{~mm}$ from the skin edge, a maximum dose of $8.42 \mathrm{~Gy}$ was recorded, with a median dose of $1.76 \mathrm{~Gy}$ (range, 0.54 to 8.42 Gy) and a mean dose of $1.91 \mathrm{~Gy}$. At $15 \mathrm{~mm}$ from the skin edge, a maximum dose of 6.58 Gy was recorded, with a median dose of $1.28 \mathrm{~Gy}$ (range, 0.44 to $6.58 \mathrm{~Gy}$ ) and a mean dose of $1.41 \mathrm{~Gy}$. Maximum dose recorded at $5 \mathrm{~mm}$ from skin edge was higher than maximum dose recorded at $15 \mathrm{~mm}$ from skin edge in every patient. As $20 \mathrm{~Gy}$ was prescribed to the applicator surface and the minimum applicator-to-skin distance was maintained at more than $1 \mathrm{~cm}$, the expected maximum skin dose was approximately $5 \mathrm{~Gy}$. In 2 patients, however, maximum doses of $5.33 \mathrm{~Gy}$ and $8.42 \mathrm{~Gy}$ were recorded; the breast volumes of these patients were $309 \mathrm{~mL}$ and $289 \mathrm{~mL}$, respectively. The breast volume/applicator diameter ratios were 8.09 and 10.38. In both cases, the tumor was located close to
Table 1. Patient and treatment characteristics

\begin{tabular}{|c|c|}
\hline Characteristic & Value \\
\hline Age (yr) & $52(32-79)$ \\
\hline \multicolumn{2}{|l|}{ Pathology } \\
\hline DCIS & $7(12.7)$ \\
\hline IDC & $38(69.1)$ \\
\hline Other & $10(18.1)$ \\
\hline \multicolumn{2}{|l|}{ Site } \\
\hline Left upper & $25(45.5)$ \\
\hline Left lower & $4(7.2)$ \\
\hline Right upper & $23(41.8)$ \\
\hline Right lower & $3(5.5)$ \\
\hline Tumor size $(\mathrm{cm})$ & $1.4(0.1-2.7)$ \\
\hline$<1$ & $17(30.9)$ \\
\hline$>1$ & $37(67.3)$ \\
\hline Unmeasurable & $1(1.8)$ \\
\hline \multicolumn{2}{|l|}{ T stage } \\
\hline Tis & $7(12.7)$ \\
\hline T1 & 40 (72.7) \\
\hline $\mathrm{T} 2$ & $8(14.5)$ \\
\hline \multicolumn{2}{|l|}{$\mathrm{N}$ stage } \\
\hline No & $51(92.7)$ \\
\hline N1 & $4(7.3)$ \\
\hline \multicolumn{2}{|l|}{ Number of positive lymph nodes } \\
\hline 0 & $51(92.7)$ \\
\hline 1 & $4(7.3)$ \\
\hline \multicolumn{2}{|l|}{ Resection margin of permanent section } \\
\hline Positive & $3(5.5)$ \\
\hline Negative & $52(94.5)$ \\
\hline \multicolumn{2}{|l|}{ Adjuvant chemotherapy } \\
\hline AC (doxorubicin/cyclophosphamide) & $15(27.2)$ \\
\hline$A C+$ paclitaxel & $1(1.9)$ \\
\hline No & 39 (70.9) \\
\hline \multicolumn{2}{|l|}{ Hormone therapy } \\
\hline Yes & 37 (67.3) \\
\hline No & $18(32.7)$ \\
\hline Interval between IORT \& EBRT (mo) & $5.7(4.4-29.1)$ \\
\hline
\end{tabular}

Values are presented as median (range) or number (\%).

DCIS, ductal carcinoma in situ; IDC, invasive ductal carcinoma; IORT, intraoperative radiotherapy; EBRT, external beam radiation therapy.

the breast margin and the distance between skin and tumor, calculated from the dose distribution curve, was $1.3 \mathrm{~cm}$ for 5.33 Gy and $0.6 \mathrm{~cm}$ for 8.42 . The patient with maximum dose of 8.42 Gy at $5 \mathrm{~mm}$ from the skin edge experienced grade 2 erythema at the spot where the maximum dose was measured (Fig. 3), although no skin reaction had been reported prior to WBI.

\section{Risk factor analysis}

The median breast volume was $567.8 \mathrm{~mL}$ (range, 157.2 to $1,634.1 \mathrm{~mL}$ ) on $\mathrm{MRI}$ images in prone position and $511.4 \mathrm{~mL}$ (range, 230.1 to $1,494.8 \mathrm{~mL}$ ) on $\mathrm{CT}$ images in supine position. The median volume of the tumor-bearing hemi-breast was $287.1 \mathrm{~mL}$ (range, 104.1 to $1,015.4 \mathrm{~mL}$ ) on MRI and $287.2 \mathrm{~mL}$ (range, 112.3 to $969.2 \mathrm{~mL}$ ) on CT. In 6 (11\%) patients, the tumor 
Table 2. Toxicity (any grade) $(n=55)$

\begin{tabular}{lcccc}
\hline \multicolumn{1}{c}{ Skin toxicity } & 1 week after IORT & 1 month after IORT & Immediately after WBI & 1 month after WBI \\
\hline Erythema (grade 1) & $0(0)$ & $0(0)$ & $30(54.5)$ & $2(3.6)$ \\
Erythema (grade 2) & $0(0)$ & $0(0)$ & $3(5.4)$ & $0(0)$ \\
Wound infection & $1(1.8)$ & $0(0)$ & $0(0)$ & $0(0)$ \\
Breast edema & $0(0)$ & $0(0)$ & $0(0)$ & $0(0)$ \\
Seroma needing aspiration & $50(90.9)$ & $28(50.9)$ & $10(18.2)$ & $\mathrm{N} / \mathrm{A}$ \\
Seroma (mL) & $15.4(0-102)$ & $5.5(0-84)$ & $1.6(0-22)$ & $\mathrm{N} / \mathrm{A}$ \\
\hline
\end{tabular}

Values are presented as number (\%) or median (range).

Table 3. Toxicity (seroma $>5 \mathrm{~mL}$ )

\begin{tabular}{|c|c|c|c|c|}
\hline & \multicolumn{2}{|c|}{ Univariate analysis } & \multicolumn{2}{|c|}{ Multivariate analysis } \\
\hline & $95 \% \mathrm{Cl}$ & p-value & $95 \% \mathrm{Cl}$ & $p$-value \\
\hline $\begin{array}{l}\text { Age } \\
\text { Applicator diameter (cm) }\end{array}$ & $0.966-1.120$ & 0.295 & $0.899-1.083$ & 0.781 \\
\hline $\begin{array}{l}\leq 3.5 \\
>3.5\end{array}$ & $1.097-124.835$ & 0.042 & $1.014-154.325$ & 0.049 \\
\hline Skin tumor distance & $0.601-3.371$ & 0.422 & $0.472-4.627$ & 0.503 \\
\hline Maximum diameter on MRI (cm) & $0.652-4.083$ & 0.296 & $0.562-5.713$ & 0.324 \\
\hline Diameter of applicator/volume of breast & $0.657-1.151$ & 0.329 & $0.615-1.299$ & 0.557 \\
\hline Maximum skin dose & $0.448-1.492$ & 0.512 & $0.476-2.205$ & 0.950 \\
\hline
\end{tabular}

$\mathrm{Cl}$, confidence interval; $\mathrm{MRI}$, magnetic resonance imaging.

was located less than $1 \mathrm{~cm}$ from adjacent skin. Among 28 patients who presented with wound seroma needing aspiration 1 month after IORT, median and mean volume of seroma were $1.5 \mathrm{~mL}$ (range, 1.0 to $84.0 \mathrm{~mL}$ ) and $5.5 \mathrm{~mL}$. According to our institution's criteria, 15 patients presented with considerable volume of seroma ( $>5 \mathrm{~mL}$ ). Analysis of factors associated with a considerable volume of seroma $(>5 \mathrm{~mL}) 1$ month after IORT revealed that applicator diameter was a significant factor in both univariate $(p=0.042)$ and multivariate analyses ( $p=$ 0.049) (Table 3). Linear regression analysis showed a significant correlation between maximum skin dose and preoperative breast volume $(p<0.001)$, distance between skin and tumor on preoperative images $(p=0.001)$, and ratio of breast volume and applicator diameter $(p<0.001)$. Table 4 summarizes the results of linear regression analysis of factors associated with skin dose.

\section{Receiver operating characteristic (ROC) analysis}

Thirty-eight patients had a maximum skin dose larger than 2 Gy, while 17 patients had a maximum skin dose larger than 3 Gy. Median and mean skin dose were 2.26 Gy (range, 1.03 to $8.42 \mathrm{~Gy}$ ) and $2.68 \mathrm{~Gy}$. The inflection point of the maximum skin dose among the 55 patients was located between $2 \mathrm{~Gy}$ and 3 Gy. Therefore, patients recording maximum skin dose higher than $3 \mathrm{~Gy}$ can be regarded as larger than average group. ROC

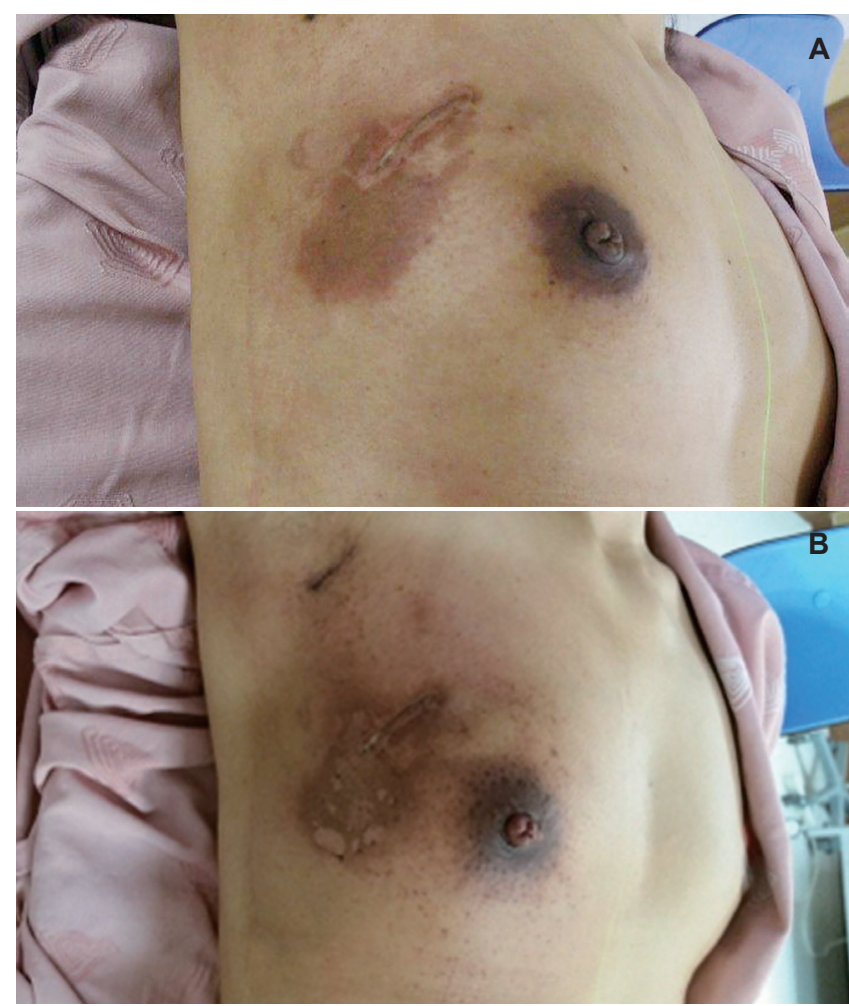

Fig. 3. Patient with maximum dose of 8.42 Gy developed skin toxicity during external beam radiation therapy (EBRT). Photographs were taken (A) after 14 fractions of EBRT and (B) immediately after the completion of EBRT. 
Table 4. Factors associated with skin dose

\begin{tabular}{lrr}
\hline & $R^{2}$ & $p$-value \\
\hline Skin-tumor distance & 0.184 & 0.001 \\
Breast volume & & \\
CT & 0.254 & $<0.001$ \\
MRI & 0.274 & $<0.001$ \\
Diameter of applicator/volume of breast & & \\
CT & 0.245 & $<0.001$ \\
MRI & 0.210 & $<0.001$ \\
Volume of applicator/volume of breast & & \\
CT & 0.060 & 0.072 \\
MRI & 0.067 & 0.060 \\
\hline
\end{tabular}

$\mathrm{CT}$, computed tomography; MRI, magnetic resonance imaging.

analysis was performed to distinguish useful parameters for predicting a maximum skin dose larger than $3 \mathrm{~Gy}$. Among the risk factors mentioned above, the ratio of applicator diameter and breast volume was the best parameter for anticipating skin dose greater than $3 \mathrm{~Gy}$. The area under the curve (AUC) was 0.820 , and a threshold value of 7 resulted in the optimal sensitivity and specificity, $82.4 \%$ and $73.4 \%$, respectively. The AUC of breast volume and the distance between skin and tumor were 0.165 and 0.267 .

\section{Discussion and Conclusion}

IORT boost is known to offer several advantages over external beam boost in patients with breast cancer undergoing BCS. Immediate initiation of radiation after tumor removal may reduce the chances of tumor cell repopulation; direct placement of the applicator in the tumor cavity could reduce the possibility of targeting error that may occur due to daily setup errors and vague identification of the tumor bed on CT images acquired several weeks after surgery. Furthermore, dose distribution with steep dose fall-off allows increased dose to the tumor bed while reducing radiation to adjacent structures including skin and other organs such as the heart and lungs $[8,9]$.

Although a few IORT-alone trials reported lower toxicity and survival rates comparable to those of WBI in low-risk patients with early breast cancer $[2,7]$, higher local recurrence rates and lack of long-term follow-up of those studies prevent clinicians from moving to a single session of IORT from WBI [10-12]. On the other hand, IORT could be a method of delivering a boost dose in combination with WBI; its feasibility as a boost treatment has been examined retrospectively. Vaidya et al. [13] reported that lumpectomy and TARGIT boost combined with WBI resulted in superior local tumor control compared to the results of the European Organization for Research and Treatment of Cancer (EORTC) boost trial or the Standardization of Breast Radiotherapy-B (START-B) trial. In addition, a single center experience at University Medical Centre Mannheim using low-kV X-ray IORT as a boost resulted in 5-year overall survival of $91.3 \%$ with 5-year local recurrence-free survival of 97.0\% [6]. An ongoing trial, TARGIT-B, is comparing IORT boost and EBRT boost in high-risk patients.

Intrabeam IORT requires inserting a spherical applicator in the tumor cavity and delivering a high radiation dose in a single fraction; thus, the toxicity risk is likely dependent on geometric variations such as applicator diameter, tumor location, and breast volume. However, no published studies clearly indicate exclusion criteria based on such geometric variables in the application of Intrabeam IORT.

As the breast volume of East Asian women is generally smaller than that of Western patients [14], it is imperative that the risk factors associated with increased toxicity are evaluated prior to the wide use of Intrabeam IORT in Korea. We conducted an in vivo dosimetry study for patients undergoing Intrabeam IORT followed by WBI, and correlated geometrical variables of the breast with increased maximum skin dose.

Some researchers regard seroma as a natural consequence of $\mathrm{PBI}$ rather than a complication [15] and have concluded that IORT is not associated with increased volume of seroma requiring treatment [16]. Even though other factors than IORT boost, such as body mass index and age could contribute to the formation of seroma [5], careful observation is necessary after IORT, as wound seroma needing aspiration was reported more frequently in patients undergoing IORT than in patients undergoing EBRT in the TARGIT-A trial as well as in the report by Kraus-Tiefenbacher et al., in which $48 \%$ of patients had measurable seroma 1 month after IORT boost $[2,5]$. Although neither of the study provided the optimum value for evaluation of seroma formation, we used $5 \mathrm{~mL}$ as a criteria considering mean volume of seroma 1 month after IORT boost was $5.5 \mathrm{~mL}$. The present study showed that the applicator diameter was an independent predictor of significant seroma formation; however, whether or not the volume of seroma also depends on the high-dose irradiation during IORT remains to be determined.

Cosmetic outcome is an important factor that should be considered in patients with breast cancer. Cracco et al. [17] reported comparable cosmetic outcomes between 81 patients treated with IORT and 105 patients treated with traditional EBRT. Further follow-up is required to assess the rate of late complications and cosmetic outcomes in Korean patients with 
breast cancer undergoing IORT boost followed by WBI.

The novelty of this study is in determining selection criteria for high-risk patients using in vivo dosimetry during IORT. Recently, OSLD has been widely used in measuring multiple radiation dose types, such as heavy ion beams, photons, electrons, and protons. Sharma and Jursinic [18] successfully used OSLD in surface dose measurement for high dose rate (HDR) brachytherapy with minimal uncertainty. The data acquired from in vivo dosimetry were useful in determining factors related to higher skin dose, which may increase the risk of acute and late skin reactions. As we have illustrated, 2 patients in the present study received skin doses higher than 5 Gy despite efforts to keep adjacent skin at least $1 \mathrm{~cm}$ away from the applicator. Therefore, patients who have similar characteristics to these 2 patients, such as relatively small breast volume, tumor location close to the breast margin, and small skin-to-tumor distance, require careful attention. In the present study, preoperative breast volume, distance between skin and tumor on preoperative images, and ratio of breast volume and applicator diameter were found to be significant factors associated with higher skin dose; these factors must be considered before performing IORT. We are now using these pre-selection criteria in order to exclude patients with a high risk of skin toxicity at our institution.

The safety of IORT alone in the Asian population was reported by Sawaki et al. [19], who evaluated the toxicity of IORT with $21 \mathrm{~Gy}$ in 5 Japanese patients with early breast cancer. The results of boost IORT in Asian patients are minimal; no institution has yet performed IORT for patients with breast cancer in Korea.

In conclusion, IORT boost was well tolerated among Korean patients with breast cancer, without severe acute toxicity. The data acquired in this study using in vivo dosimetry could be useful in selecting appropriate candidates for IORT boost in the future. The results of the phase 2 trial and the assessment of late toxicity will follow.

\section{Conflict of Interest}

No potential conflict of interest relevant to this article was reported.

\section{Acknowledgments}

This work was supported by KOSRO Young Investigator Fund, new faculty research seed money grant of Yonsei University College of Medicine for 2016 (2016-32-0018), and Technology
Commercialization Program through Innopolis Foundation of Korea funded by the Ministry of Science, ICT and Future Planning (ACC-2016-DDI-00793).

\section{References}

1. Njeh $C F$, Saunders MW, Langton CM. Accelerated partial breast irradiation (APBI): a review of available techniques. Radiat Oncol 2010;5:90.

2. Vaidya JS, Joseph DJ, Tobias JS, et al. Targeted intraoperative radiotherapy versus whole breast radiotherapy for breast cancer (TARGIT-A trial): an international, prospective, randomised, non-inferiority phase 3 trial. Lancet 2010;376:91102.

3. Smith BD, Arthur DW, Buchholz TA, et al. Accelerated partial breast irradiation consensus statement from the American Society for Radiation Oncology (ASTRO). Int J Radiat Oncol Biol Phys 2009;74:987-1001.

4. Chua BH, Henderson MA, Milner AD. Intraoperative radiotherapy in women with early breast cancer treated by breast-conserving therapy. ANZ J Surg 2011;81:65-9.

5. Kraus-Tiefenbacher $U$, Bauer L, Kehrer T, Hermann B, Melchert F, Wenz F. Intraoperative radiotherapy (IORT) as a boost in patients with early-stage breast cancer: acute toxicity. Onkologie 2006;29:77-82.

6. Blank E, Kraus-Tiefenbacher U, Welzel G, et al. Single-center long-term follow-up after intraoperative radiotherapy as a boost during breast-conserving surgery using low-kilovoltage x-rays. Ann Surg Oncol 2010;17 Suppl 3:352-8.

7. Veronesi $U$, Orecchia $R$, Luini $A$, et al. Intraoperative radiotherapy during breast conserving surgery: a study on 1,822 cases treated with electrons. Breast Cancer Res Treat 2010;124:141-51.

8. Benda RK, Yasuda G, Sethi A, Gabram SG, Hinerman RW, Mendenhall NP. Breast boost: are we missing the target? Cancer 2003;97:905-9.

9. Holmes DR. Intraoperative radiotherapy in breast conserving surgery. J Surg Oncol 2014;110:68-74.

10. Silverstein MJ, Fastner G, Maluta $S$, et al. Intraoperative radiation therapy: a critical analysis of the ELIOT and TARGIT trials. Part 1. ELIOT. Ann Surg Oncol 2014;21:3787-92.

11. Hepel J, Wazer DE. A flawed study should not define a new standard of care. Int J Radiat Oncol Biol Phys 2015;91:255-7.

12. Silverstein MJ, Fastner G, Maluta S, et al. Intraoperative radiation therapy: a critical analysis of the ELIOT and TARGIT trials. Part 2. TARGIT. Ann Surg Oncol 2014;21:3793-9.

13. Vaidya JS, Baum M, Tobias JS, et al. Long-term results of 
targeted intraoperative radiotherapy (TARGIT) boost during breast-conserving surgery. Int J Radiat Oncol Biol Phys 2011;81:1091-7.

14. Anderson JD, Chandler SC, Mason MA, et al. Scientific analysis reveals major differences in the breast size of women in different countries. J Female Health Sci [Internet]. JFH. TD.13.098 [cited 2017 May 25]. Available from: http://www. sciencedatabaseonline.org/ADB1/Scientific\%20Article\%20 JOFHS.pdf.

15. Patel PS, Yan W, Trichter $S$, et al. Seroma is an expected consequence and not a complication of MammoSite brachytherapy. Breast J 2011;17:498-502.

16. Kraus-Tiefenbacher U, Welzel G, Brade J, et al. Postoperative seroma formation after intraoperative radiotherapy using lowkilovoltage $X$-rays given during breast-conserving surgery. Int J Radiat Oncol Biol Phys 2010;77:1140-5.

17. Cracco S, Semprini G, Cattin F, et al. Impact of intraoperative radiotherapy on cosmetic outcome and complications after oncoplastic breast surgery. Breast J 2015;21:285-90.

18. Sharma $R$, Jursinic PA. In vivo measurements for high dose rate brachytherapy with optically stimulated Iuminescent dosimeters. Med Phys 2013;40:071730.

19. Sawaki $M$, Kondo $N$, Horio $A$, et al. Feasibility of intraoperative radiation therapy for early breast cancer in Japan: a singlecenter pilot study and literature review. Breast Cancer 2014;21:415-22. 